\title{
Wnt5a Is Necessary for Normal Kidney Development in Zebrafish and Mice
}

\author{
Liwei Huang a An Xiao ${ }^{\text {a }}$ Soo Young Choid ${ }^{d}$ Quane Kan ${ }^{\text {a }}$ Weibin Zhou ${ }^{c}$ \\ Maria F. Chacon-Heszele ${ }^{f}$ Yun Kyoung Ryu ${ }^{b}$ Sarah McKenna $^{f}$ Xiaofeng Zuo $^{d}$ \\ Rejji Kuruvillab Joshua H. Lipschutz ${ }^{\text {d, e }}$ \\ ${ }^{a}$ Department of Medicine, Eastern Virginia Medical School, Norfolk, Va., ${ }^{b}$ Department of Biology, Johns Hopkins \\ University, Baltimore, Md., 'Department of Pediatrics and Communicable Disease, University of Michigan, \\ Ann Arbor, Mich., 'Department of Medicine, Medical University of South Carolina, ${ }^{\text {e Department }}$ of Medicine, \\ Ralph H. Johnson Veterans Affairs Medical Center, Charleston, S.C., and fDepartment of Medicine, University of \\ Pennsylvania, Philadelphia, Pa., USA
}

\section{Key Words}

Wnt5a - Kidney development · Pronephros · Mesonephros . Metanephros

\begin{abstract}
Background: Wnt5a is important for the development of various organs and postnatal cellular function. Little is known, however, about the role of Wnt5a in kidney development, although WNT5A mutations were identified in patients with Robinow syndrome, a genetic disease which includes developmental defects in kidneys. Our goal in this study was to determine the role of Wnt5a in kidney development. Methods: Whole-mount in situ hybridization was used to establish the expression pattern of Wnt5a during kidney development. Zebrafish with wnt5a knockdown and Wnt5a global knockout mice were used to identify kidney phenotypes. Results: In zebrafish, wnt5a knockdown resulted in glomerular cyst formation and dilated renal tubules. In mice, Wnt5a global knockout resulted in pleiotropic, but severe, kidney phenotypes, including agenesis, fused kidney, hydronephrosis and duplex kidney/ureter. Conclusions: Our data demonstrated the important role of Wnt5a in kidney development. Disrupted Wnt5a resulted in kidney cysts in zebrafish and pleiotropic abnormal kidney development in mice. @ $2014 \mathrm{~S}$. Karger AG, Basel
\end{abstract}

\section{KARGER}

(c) 2014 S. Karger AG, Basel

$1660-2129 / 14 / 1282-0080 \$ 39.50 / 0$

E-Mail karger@karger.com

www.karger.com/nee

\section{Introduction}

Congenital kidney and urinary tract abnormalities have been described in patients with Robinow syndrome $[1,2]$, but the molecular mechanisms are unclear. WNT5A mutations were found in patients with Robinow syndrome and $W n t 5 a$, and its receptor Ror 2 knockout mice show phenotypes similar to these patients [3]. Wnt5a is a noncanonical glycoprotein of the Wnt family that regulates a wide range of developmental processes. It is known that Wnt5a is essential for proper skeletal, urogenital and gonad development $[4,5]$, but the role of Wnt5a in kidney development is unknown.

The mammalian kidney originates from intermediate mesoderm (IM). The metanephros, which develops into the permanent kidney, forms when the ureteric bud (UB) grows out of the nephric (wolffian) duct (ND) and contacts the metanephric mesenchyme (MM). The UB then elongates and branches to form the collecting duct system. The metanephric mesenchymal cells condense around the tip of the UB, aggregate, epithelialize and differentiate to podocytes and proximal and distal tubular cells.

Our goal in this study was to determine the role of Wnt5a in kidney development. We used two models, ze- 
Fig. 1. $w n t 5 a$ expression localizes in the zebrafish kidney. Lateral view (a) and dorsal view (b) of wmISH of zebrafish embryos at $72 \mathrm{hpf}$ with the staining of wnt5a probes. The Sense probe (upper panel in a) shows no signal. c Dorsal view of zebrafish embryos at $72 \mathrm{hpf}$ with the wt1a antisense probe, used as a positional marker, showing wt1a expression in the pronephric glomerulus. Wnt5a is expressed in the zebrafish pronephric glomerulus (arrow). Wnt5a can also be seen in the proximal tubules in the lateral view (a, arrowhead), but not in the dorsal view (b).

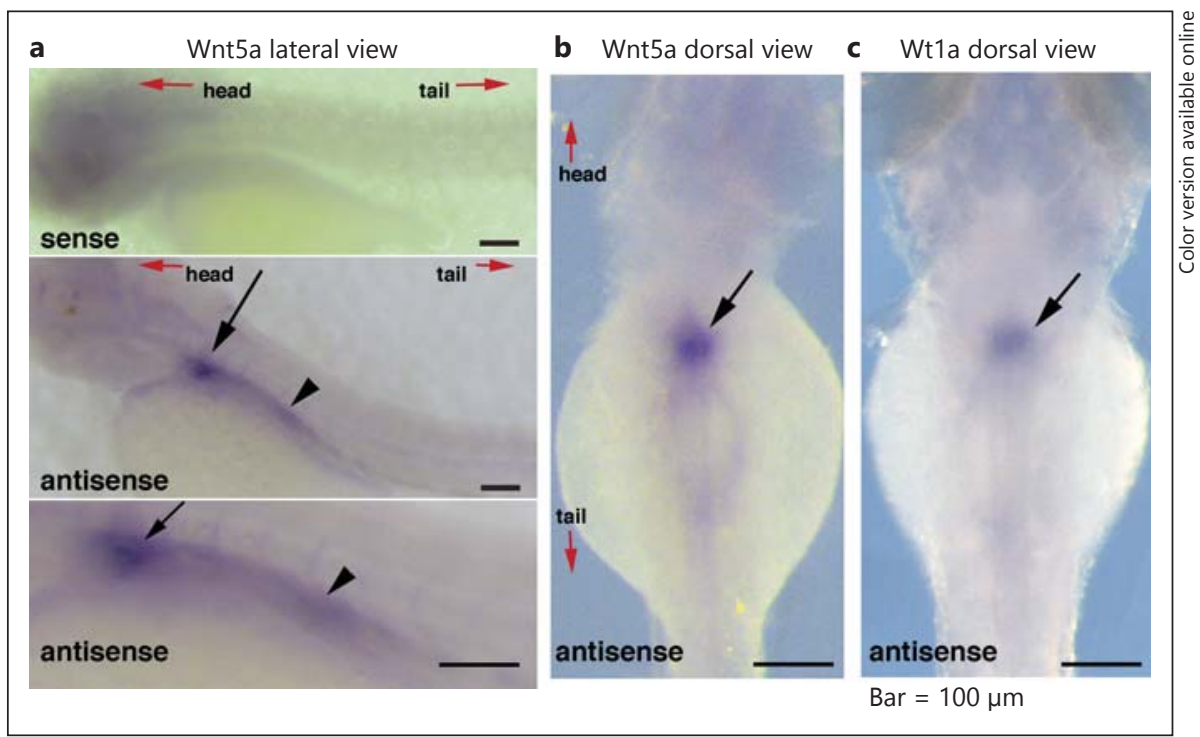

brafish and mice. The simple nature of the zebrafish pronephric kidney makes it a suitable system to study the early developmental events that lay the foundation for genesis of more complex kidneys, while mouse metanephric kidney development is similar to human kidney development.

\section{Methods}

\section{Zebrafish Maintenance}

Wild-type and $T g(w t 1 b: G F P)$ (green fluorescent protein) transgenic zebrafish [6] were reared and maintained as described [7]. Embryos were collected after natural spawn, kept at $28.5^{\circ} \mathrm{C}$, and staged as described [8]. Embryos from 48-hpf $T g(w t 1 b: G F P)$ zebrafish were anesthetized in $0.016 \%$ tricaine solution and embedded in 3\% methyl cellulose with dorsal side facing up and imaged using a fluorescence microscope (Leica M205C).

\section{Zebrafish Injections and Morpholino Oligonucleotides}

Wnt 5 a morpholino oligonucleotides (MOs) were generated by Gene Tools, LLC (Philomath, Oreg., USA). MOs were injected into embryos at the one-to-four-cell stage as described [9]. Capped mouse $W n t 5 a$ full-length mRNA was synthesized using the mMessage mMachine T7 kit (AM1344, Ambion). For the rescue experiments, 40 pg of Wnt $5 a$ mRNA was coinjected with the AUG-MOs into one-to-four-cell stage embryos.

Wnt5a $a^{-/-}$Null Mice

$W n t 5 a^{-/-}$null mice were generated by mating CMV-Cre mice (Jackson Lab stock No. 006054) with Wnt5a fl/fl mice [10] and were similar in phenotype to the commercially available $W n t 5 a$ global knockout mice [11]. The day of vaginal plug was considered E0.5. The $W n t 5 a^{-/-}$embryos were identified visually by a reduced size phenotype, and the absence of Wnt5a was confirmed by PCR as described $[10,11]$. IM from E10.5, E11.5, and metanephros from
E13.5 and E15.5 were dissected out and fixed for whole-mount in situ hybridization (wmISH). E11.5-18.5 metanephros were dissected out, and images were taken with a Leica M205C dissecting microscope. MRI of E16.5 Wnt5a $a^{-/-}$mouse embryos was performed at the Penn Small Animal Imaging Facility using a 9.4-tesla $(400 \mathrm{MHz})$ vertical bore spectrometer with $55-\mathrm{mm}$ gradients.

\section{Whole-Mount in situ Hybridization}

Mouse Wnt5a was subcloned from MSCV2.2Wnt5aIRES-GPF (kindly provided by Dr. Stephen Jones at University of Massachusetts Medical School) into the pEGM-T Easy Vector (Promega). The plasmid for the mouse Ret gene was kindly provided by Dr. Mendelsohn, Columbia University. The zebrafish wnt5a template was prepared by TA cloning. Digoxigenin-labeled RNA probes were prepared by in vitro transcription (DIG RNA Labeling kit, Roche). wmISH in mouse or zebrafish embryos was performed as previously described $[5,12]$.

\section{Statistical Analyses}

Phenotypes for the rescue experiments were classified as abnormal after MO injection by the presence of any combination of the following features: edema, short body size and curled tails. For comparison of means, a t-test was performed using SPSS software (v.15.0; SPSS Inc., Chicago, Ill., USA). For all tests, $\mathrm{p}<0.05$ was considered to represent statistically significant differences.

\section{Results}

\section{Wnt5a Localizes to the Developing Zebrafish Kidney} wmISH of wild-type zebrafish embryos at $72 \mathrm{~h}$ after fertilization (hpf) confirmed $w n t 5 a$ expression in the pronephric kidney (fig. 1a, b). The wtla probe served as a positional marker to confirm wnt5a expression in the pronephric glomerulus (fig. 1c). 


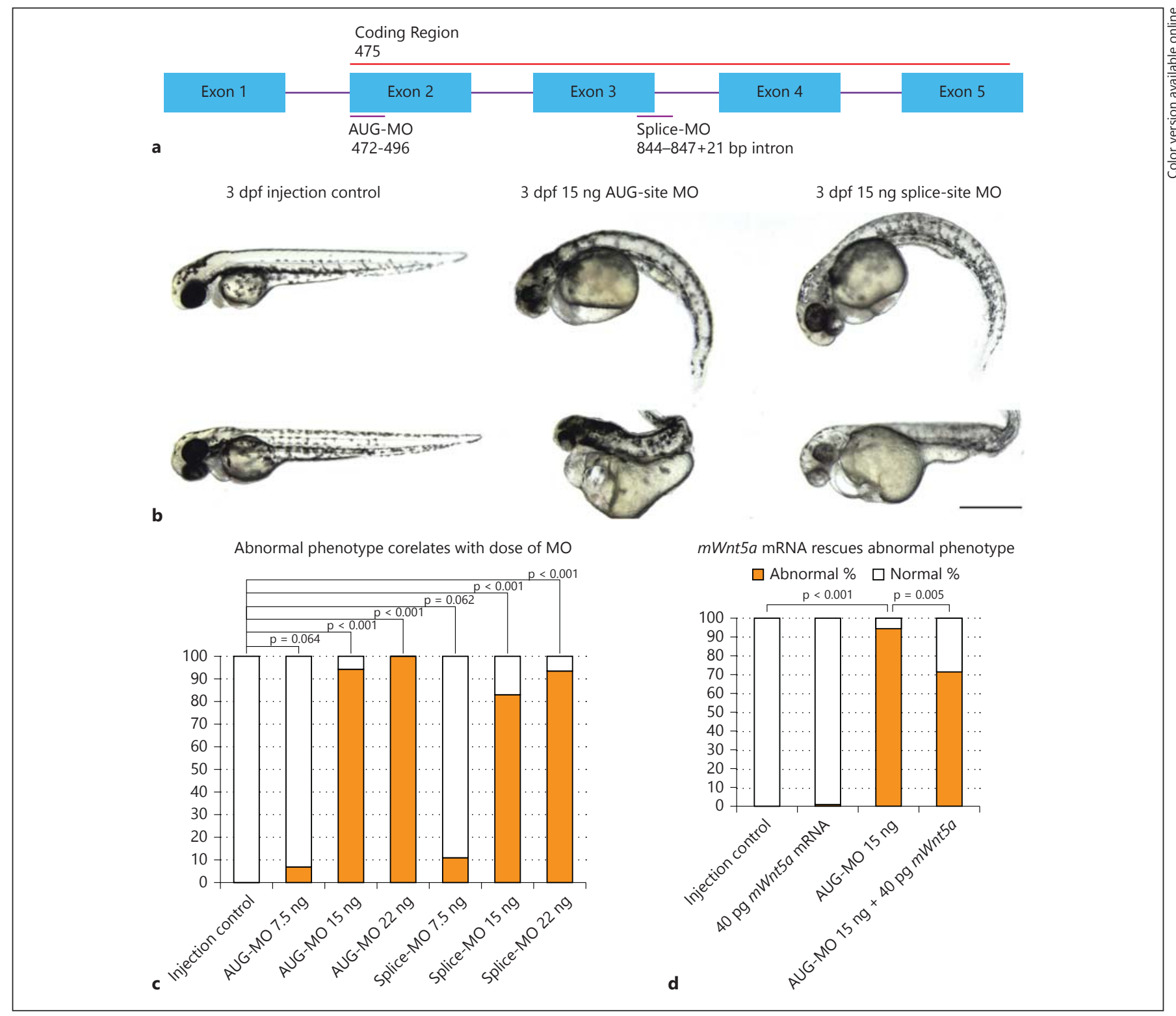

Fig. 2. $w n t 5 a$ knockdown by antisense morpholinos results in abnormal body curvature. a Diagram showing the zebrafish $w n t 5 a$ gene, the coding region, and the regions targeted by the AUG- and splice-MO. The sequences of MOs complementary to the gene target were: AUGMO: 5'-ACTTCAGCTTCAGCAGCATCATAAC-3'(GI_BC150218) and splice-MO: $\quad 5^{\prime}$-gtgtttttgcggcgtacatacCGAT-3'(GI_BC150218; capitalized letters: targeted exon; non-capitalized letters: intron). b Phenotype of injection control (phenol red) embryos and AUG- and splice-site $w n t 5 a$ morphants at $3 \mathrm{dpf}$ with dorsal to the top and anterior to the left. The AUG- and splice-site morphants phenocopied each other with a downward curved tail, small eyes, and pericardial edema. 'Class 1' morphants exhibits a 'curly tail down' body shape and pericardial edema (90\% of total abnormal morphants, first row middle and right column). 'Class 2' morphants exhibits more severe defects including a reduced and abnormally shaped body axis and significant pericardial edema ( $10 \%$ of total abnormal morphants, second row middle and right column). $\mathrm{Bar}=2 \mathrm{~mm}$. c A dose-response occurs, with increasing amounts of morpholino resulting in a worsening phenotypes for both the AUG- and splice-MOs ( $p=0.064$ in AUG-MO $7.5 \mathrm{ng}$ vs. control, $\mathrm{p}<0.001$ in AUG-MO 15 ng vs. control, $\mathrm{p}<0.001$ in AUG-MO 22 ng vs. control, $\mathrm{p}=0.062$ in splice-MO $7.5 \mathrm{ng}$ vs. control, $\mathrm{p}<0.001$ in splice-MO $15 \mathrm{ng}$ vs. control, $\mathrm{p}<0.001$ in splice-MO $22 \mathrm{ng}$ vs. control). The use of two MOs with unrelated sequences demonstrates the specificity of the observed phenotype. $\mathbf{d}$ The AUG-MO phenotype can be partially rescued with mouse Wnt $5 a$ mRNA, which is resistant to the AUG-MO due to a difference in primary base pair structure, thereby demonstrating that the phenotypes are not due to off-target effects. Abnormal phenotypes as described above are counted at $72 \mathrm{hpf}$ in different injection groups (we tried mouse mRNA dose from 20 to $120 \mathrm{pg}$ and identified $40 \mathrm{pg}$ RNA to be the dose at which nonspecific or toxic effects of the RNA do not occur). The difference is statistically significant at $\mathrm{p}<0.05$ ( $\mathrm{p}<0.001$ in AUG-MO $15 \mathrm{ng}$ vs. control group, $\mathrm{p}=0.005$ in AUG-Mo $15 \mathrm{ng}+40 \mathrm{pg} m W n t 5 a$ mRNA group vs. AUG-MO 15 ng group). 


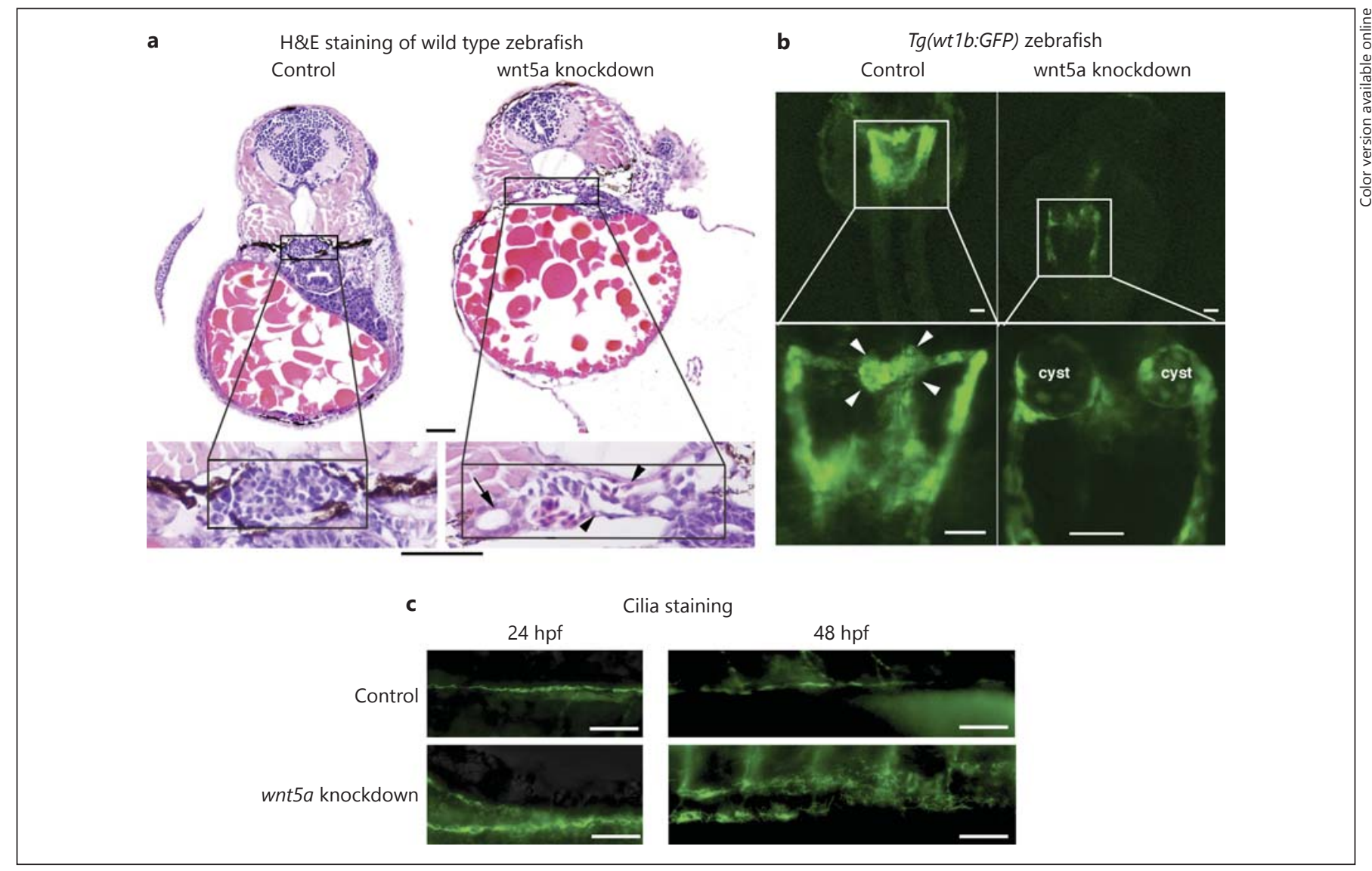

Fig. 3. $w n t 5 a$ knockdown by antisense morpholinos results in pronephric cyst formation. a HE staining of the transverse histological sections of 72-hpf morphant embryos reveals disrupted glomerular structures and dilated glomeruli (arrowhead) and renal tubules (arrow) after $w n t 5 a$ knockdown by both AUG- and splice-MOs (image is from control and AUG-injected zebrafish; splice-MO injection showed similar results, but image is not shown here). Bar $=20$ $\mu \mathrm{m}$. b Representative images of the pronephros of 48-hpf

\section{Wnt5a Is Necessary for Zebrafish Pronephros}

Development

We knocked down wnt5a with a translation blocking morpholino (AUG-MO), which targets the start codon and affects both maternal and zygotic wnt5a mRNA, and an exon/intron border splice morpholino (splice-MO), which targets the third splice donor site and affects only the zygotic transcript of wnt5a (fig. 2a). Both the AUG and the splice morphants phenocopied each other, with multiple defects at $72 \mathrm{hpf}$, including: reduced axis length, curly tail down body axis, and pericardial edema (fig. 2b). HE staining of the transverse histological sections of morphants revealed disrupted and dilated glomerular structure and dilated proximal tubules (fig. 3a). The $T g(w t 1 b: G F P)$ transgenic zebrafish [7], in which GFP is driven by the $w t 1 b$
$\mathrm{Tg}(w t 1 b: G F P)$ transgenic zebrafish embryos injected with phenolred control and wnt5a AUG-MO. Cystic glomeruli are clearly visible in the wnt5a morpholino-injected animals but not in the control (phenol-red) ones. Arrowheads indicate glomeruli. (SpliceMO injection showed similar results, which is not shown here.) Bar $=50 \mu \mathrm{m}$. $\mathbf{c}$ Representative images of the ciliary staining in pronephros at 24 and $48 \mathrm{hpf}$. wnt5a knockdown results in disordered ciliary structure compared with injection control. Bar $=50 \mu \mathrm{m}$.

promotor so that GFP recapitulates endogenous expression of $w t 1 b$, was used to examine the pronephric structure. The $T g(w t 1 b: G F P)$ fish showed glomerular cyst formation after wnt5a knockdown (fig. 3b). Cilia staining showed disordered pronephric cilia in wnt5a knockdown zebrafish compared to the injection controls (fig. 3c).

\section{Wnt5a Localizes to the Developing Mouse Kidney}

To determine whether Wnt5a plays a role in mouse metanephric kidney development, we performed wmISH of Wnt5a in E10.5 and E11.5 IM, and E13.5 and E15.5 metanephros. wmISH of the Ret gene expression was used to identify the ND and UB structure (fig. 4a-d). At E10.5, Wnt5a mRNA was diffusely expressed in the entire IM with higher expression level caudally (fig. 4e, g). At 

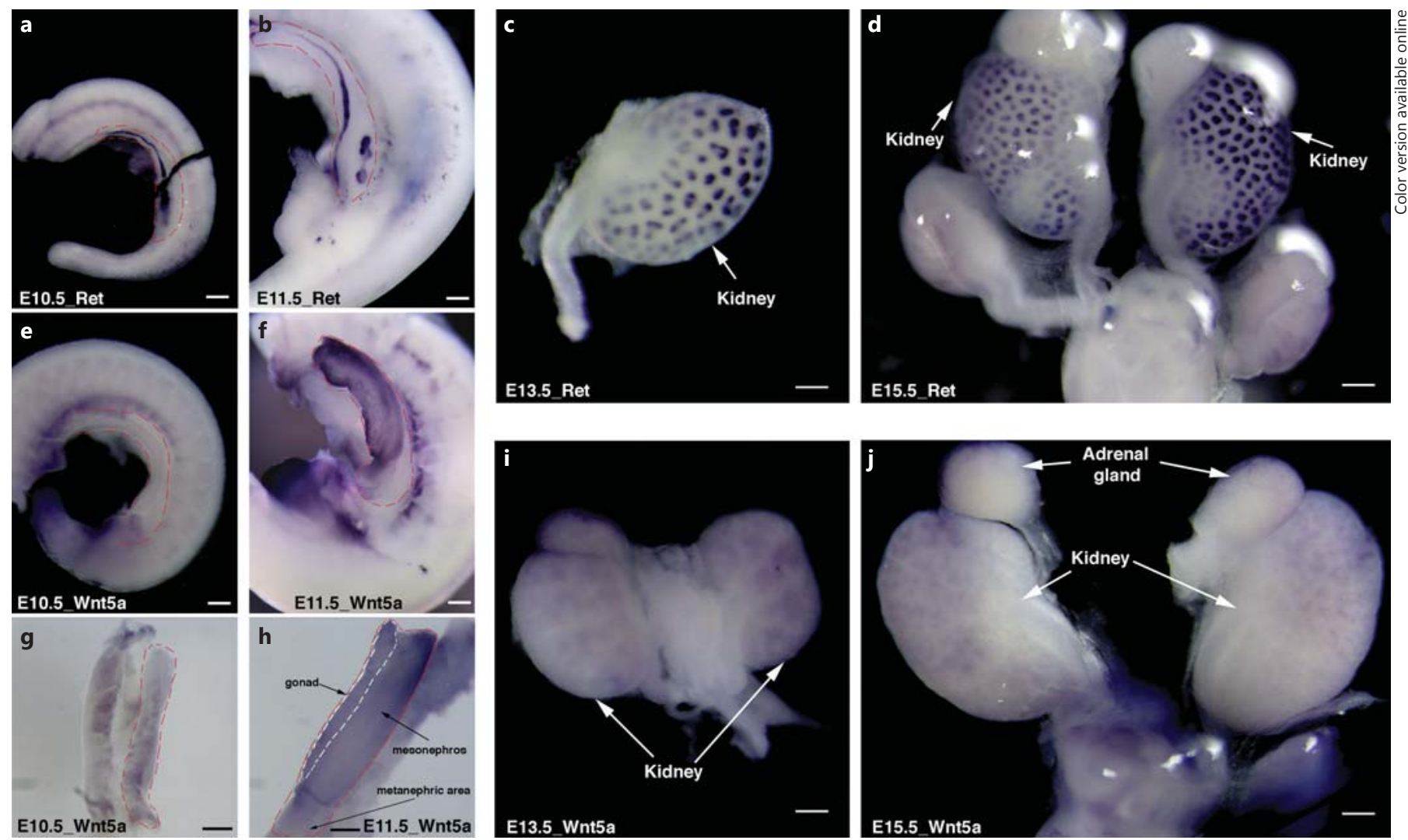

Fig. 4. wmISH demonstrates $W n t 5 a$ expression in the mouse IM, mesonephros and metanephric kidney. wmISH of Ret gene expression (a-d) is used to identify IM and UB location at E10.5 (a), E11.5 (b), E13.5 (c) and E15.5 (d). Red dashed lines outline the IM tissues. At E10.5, while Ret is expressed in the ND and at the site of UB outgrowth (a), Wnt5a is diffusely expressed at a lower level

Fig. 5. Metanephric kidney development is severely compromised in $W n t 5 a$ global knockout mice as determined by MRI, fine dissection, and HE staining. a, b MRI of E16.5 mouse embryos. A normal-sized kidney is seen in the wild-type mouse embryo (a), whereas a $W n t 5 a^{-/-}$mouse embryo shows absence of kidney formation (b). Due to the skeletal abnormalities, it was difficult to identify identical sections in control and Wnt5a $a^{-/}$ mice. c-f Wild-type and Wnt5 $a^{-1-}$ mouse kidneys (when tissue was present) and urinary tracts were dissected at E16.5. The kidneys from $W n t 5 a^{-/-}$mutants shows pleiotropic, but severe, kid-

in the entire IM region with higher expression caudally (e and $\mathbf{g}$ ). $\mathbf{f}, \mathbf{h}$ At E11.5, Wnt5a expression level increases significantly in the gonad and mesonephric kidney, but not in the metanephric region where the UB initiates. At E13.5 (i) and E15.5 (j), Wnt5a is expressed in the metanephric kidney, with a pattern similar to Ret expression. Bar $=200 \mu \mathrm{m}$.

ney phenotypes, including bilateral kidney agenesis (c), unilateral kidney agenesis (d), fused kidneys (e) and hydroureteronephrosis (f). c-f Bar $=1 \mathrm{~mm}$. g HE staining of the embryonic kidneys from wild-type and $W n t 5 a^{-/-}$mice. Histologic sections of E16.5 wild-type (left) and $W n t 5 a^{-/-}$(right) embryonic kidneys shows markedly reduced kidney tubules and glomeruli in $W n t 5 a^{-/-}$embryos. There is also significant dilatation of the renal tubules and hydronephrosis in the $W n t 5 a^{-/-}$mutant embryos. g Bar $=50 \mu \mathrm{m}$.

(For figure see next page.)

E11.5 when the UB starts to branch, Wnt5a expression increased significantly in the gonad and mesonephric region, however, not in the metanephric region where the UB starts to initiate (fig. 4f, h). At E13.5 and E15.5, Wnt5a was expressed in the metanephros (fig. $4 \mathrm{i}, \mathrm{j}$ ) with a pattern similar to Ret expression (fig. 4c, d), indicating uretic bud expression of $W n t 5 a$.
Wnt5a $a^{-/-}$Mice Have Abnormal Kidney Development

$W n t 5 a^{-1-}$ mice were used to assess the loss of Wnt5a expression on kidney development. Since $W n t 5 a^{-/-}$mice are perinatal lethal, an MRI was performed on E16.5 embryos, and showed kidneys in the wild-type mice (fig. 5a), but not in the Wnt5a-l- embryo (fig. 5b). We then dissected multiple wild-type and $W n t 5 a^{-/-}$embryos and 

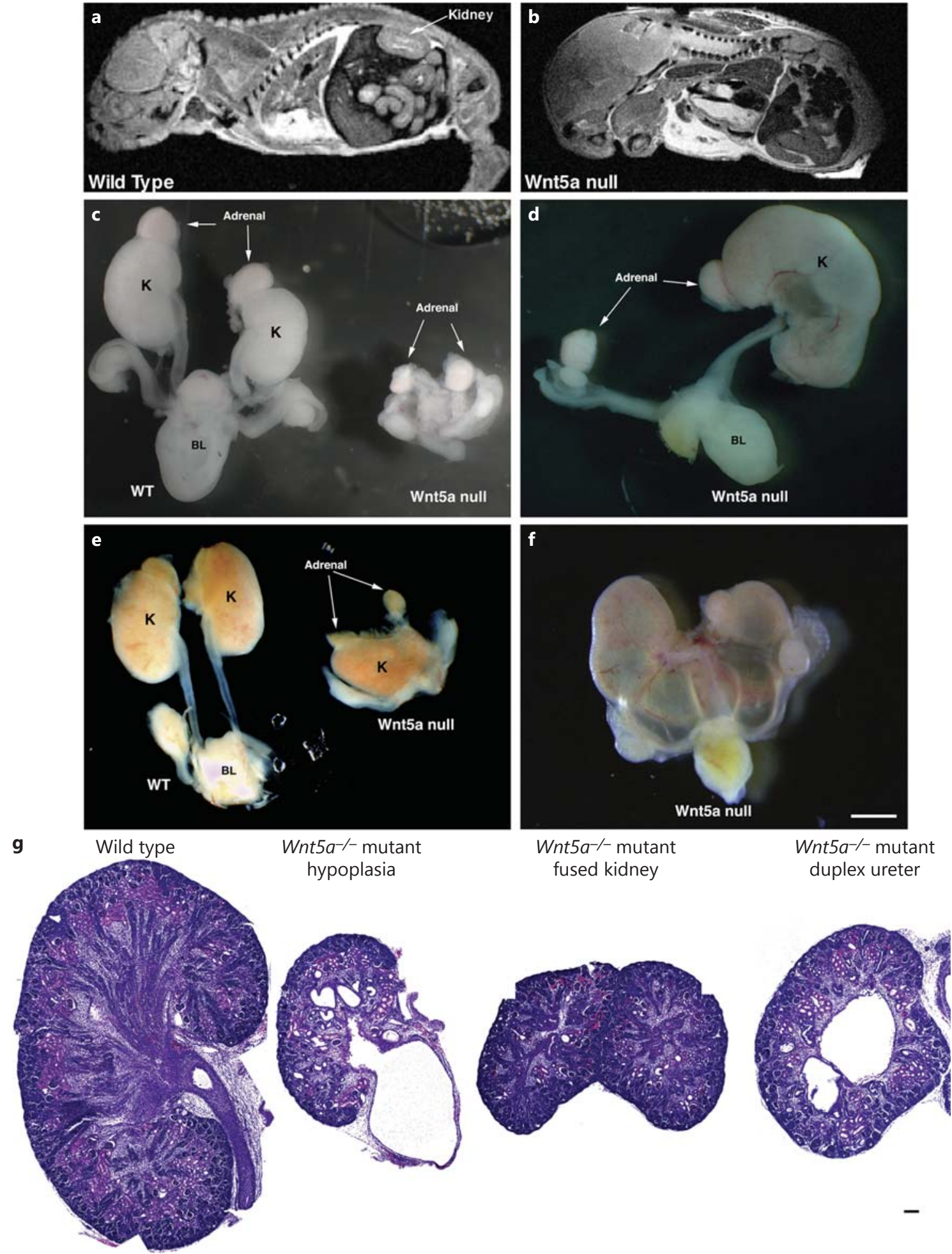
Table 1. Percentage of abnormal kidney phenotypes in Wnt5a knockout embryos

\begin{tabular}{ll}
\hline Kidney phenotype & Percentage \\
\hline Bilateral kidney agenesis & 21 \\
Unilateral kidney agenesis & 28 \\
Hydronephrosis & 71 \\
Hydroureterosis & 50 \\
Duplex kidney & 14 \\
Duplex ureter & 29 \\
Fused kidney & 36 \\
\hline
\end{tabular}

identified several different, but severe, phenotypes (fig. $5 \mathrm{c}-\mathrm{f}$, online suppl. fig. 1; for all online suppl. material, see www.karger.com/doi/10.1159/000368411, and table 1). Histology (fig. 5g) of the Wnt5a ${ }^{-/-}$kidneys showed markedly reduced kidney development.

wmISH of the Ret gene was used to identify the ND and UB structure. In wild-type embryos, UB initiated at the caudal region of the IM, and started to branch at E11.5 (fig. 6a, d). The mutant embryos had truncated IM and abnormal UB initiation, such as medial dislocation of the outgrowth sites (fig. 6e), delayed UB branching (fig. 6e) and outgrowth at more than two sites (fig. 6c, f). ByE15.5, the Ret expression was localized at the branched UB (fig. 6h); unilateral hypoplasia and duplex kidney and ureter were seen in $W n t 5 a^{-/-}$mutant embryos (fig. 6i, j).

\section{Discussion}

Pleotropic kidney defects, including cystic kidney disease [2], hypoplastic kidney [13], hydronephrosis and nephrocalcinosis [14] have been reported in patients with Robinow syndrome. Wnt5a has been found to play important roles in these patients' craniofacial and skeletal abnormalities. Our study utilized two different animal models, zebrafish and mouse, to demonstrate the role of Wnt5a during normal kidney development, and to help identify the causes of kidney abnormalities in Robinow syndrome patients.

Wnt5a knockdown in zebrafish resulted in glomerular cyst formation. Wnt5a functions in planar cell polarity (PCP) regulation in mice [15], and disruption in the PCP pathway has been shown to result in kidney cyst formation [16]. The abnormal body axis curvature with downward-curving tail in our wnt $5 a$ morphants is also seen in zebrafish with defects in cilia motility $[17,18]$ or assembly
[19]. Wnt5a morphants had disordered pronephric cilia, suggesting that wnt5a might work through ciliogenesis and PCP to control normal kidney development. It is also possible that the pronephric tubular dilation could have played a role in the ciliary phenotype. Further investigations will be conducted to establish the relationship among wnt5a, cilia and PCP.

We found pleiotropic abnormal renal phenotypes in Wnt5 $a^{-/-}$mutant mice (table 1 ). There are many examples of pleiotropic renal phenotypes in knockout mice. For instance, only one third of newborn Ret knockout mice showed complete absence of ureters and kidneys, and one tenth had bilateral kidney rudiments [20].

The cause of the duplex kidney and ureter was likely due to Wnt5a controlling IM extension [21] and regulating the interaction of ND and MM [22]. Besides duplex ureter/kidney, our data also demonstrated bilateral and unilateral kidney agenesis in $W n t 5 a$ knockout mice. Our Wnt5a knockout mice had truncated IM, medial dislocation of the UB outgrowth sites and delayed UB branching. Dislocated UB outgrow sites can cause a dysregulated interaction between ND and MM and result in kidney agenesis. Delayed UB branching results in kidney hypoplasia. It is interesting that Wnt5a strongly expresses at the mesonephros, but does not strongly express in the UB outgrowth area, indicating an important role of the mesonephros in the development of metanephros. Wnt5a may play a role in UB branching morphogenesis, but is likely not involved in the mesenchymal epithelial transformation or S-shaped body cell differentiation because these structures develop relatively normally in the $W n t 5 a$ null kidneys. Another notable phenotype of $W n t 5 a$ global knockout mice is fused kidneys. The fused kidney is secondary to defects in capsule formation, which prevents the kidneys from detaching from the body wall. The reason why loss of Wnt5a expression results in fused kidneys is not clear, though the medial dislocation of the UB outgrowth sites might be one of the causes of fused kidneys. FoxD1, a transcription factor expressed in the stromal mesenchymal region of the developing kidney, plays a role in controlling cellularity in the renal capsule [23]. Whether Wnt5a works through a Fox signaling pathway or is downstream of FoxD1 signaling remains to be seen.

Wnt5a regulates skeletal morphogenesis, and MRI of the mouse embryo showed significant skeletal abnormalities. Our previous work showed that $W n t 5 a^{-/-}$mice had significant urogenital abnormalities, which may be the reason for the hydronephrosis phenotype seen in the kidney. It is difficult to determine cyst formation in this Wnt5a mutant model because of perinatal lethality and
Huang et al. 

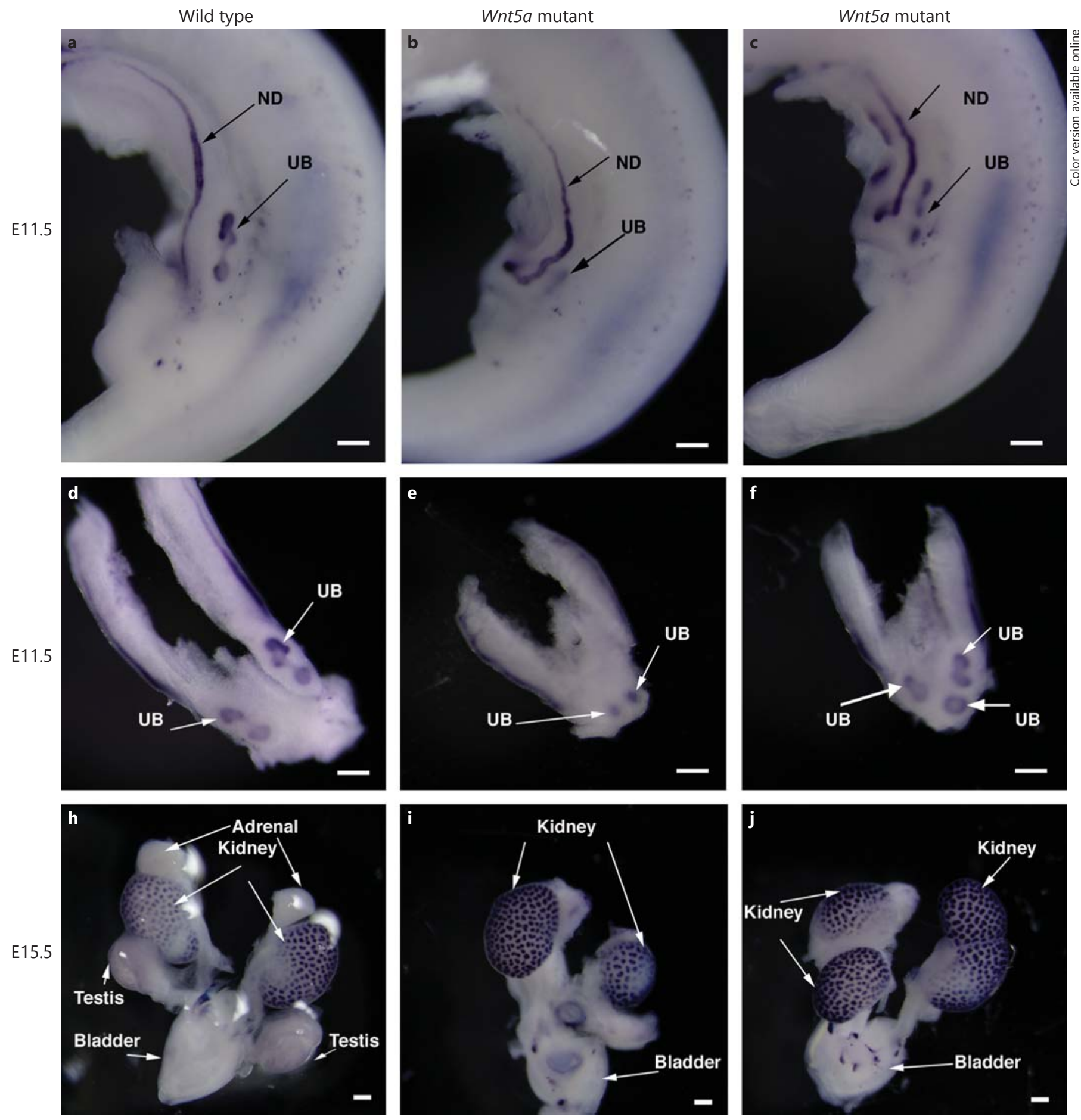

Fig. 6. wmISH of Ret gene expression in wild-type and Wnt5a knockout mouse embryos. a, d At E11.5, the UB forms at the metanephric region and starts to branch in the wild-type embryo. b, e In some Wnt5a knockout embryos, the regions containing Ret-expressing IM are limited, the site of UB outgrowth occurred at the medial region and the branching is delayed. $\mathbf{c}, \mathbf{f}$ In other Wnt5a knockout embryos, there are additional UB outgrowth sites. At E15.5, some Wnt5a knockout embryos developed unilateral kidney hypogenesis (i), while others have duplex kidneys/ure$\operatorname{ter}(\mathbf{j})$. 
urogenital abnormalities; therefore, kidney-specific Wnt5a knockout mice are needed, which we are currently generating.

In summary, the present study establishes a key role for Wnt5a during early kidney development, and allows it to join the large group of Wnts that are involved in different stages of metanephrogenesis. Whether Wnt5a is involved in ciliogenesis and PCP signaling needs further investigation. Our finding helps us to understand the mechanism of kidney and urinary anomalies in patients with Robinow syndrome [1,3]. Interestingly, defective $w n t 5 a$ signaling causes renal cyst formation in our zebrafish model and cystic kidney disease has also been reported in patients with Robinow syndrome [24]. Our future efforts will be directed towards studying kidney-specific Wnt5a knockout mice, which should allow the mice to survive long enough to fully manifest renal defects.

\section{Acknowledgements}

This study was funded by Veteran Affair Merit Award to J.H.L, NIH (DK069909 and DK047757) to J.H.L, DK093625 to L.H, Satellite Healthcare (Norman S. Coplon Extramural Research Grant) to J.H.L, and University of Pennsylvania Translational Medicine Institute (Pilot Grant) to J.H.L.

The University of Pennsylvania Biomedical Imaging Core Facility of the Cancer Center is acknowledged for providing imaging services, and the University of Pennsylvania Zebrafish Core for providing essential services such as maintenance and breeding of fish. Dr. F. Perry Wilson is gratefully acknowledged for assistance with statistical analyses. Mrs. Andrea Wecker and Dr. Michael Solhaug are gratefully acknowledged for reviewing the paper and offering helpful comments and suggestions.

\section{Disclosure Statement}

There are no competing financial interests.

\section{References}

1 Tufan F, Cefle K, Turkmen S, et al: Clinical and molecular characterization of two adults with autosomal recessive Robinow syndrome. Am J Med Genet A 2005;136A:185-189.

$\checkmark 2$ Wiens L, Strickland DK, Sniffen B, et al: Robinow syndrome: report of two patients with cystic kidney disease. Clin Genet 1990;37: 481-484.

3 Person AD, Beiraghi S, Sieben CM, et al: WNT5A mutations in patients with autosomal dominant Robinow syndrome. Dev Dyn 2010;239:327-337.

4 Maeda K, Kobayashi Y, Udagawa N, et al: Wnt5a-Ror2 signaling between osteoblastlineage cells and osteoclast precursors enhances osteoclastogenesis. Nat Med 2012;18: 405-412.

5 Huang L, Pu Y, Hu WY, et al: The role of Wnt5a in prostate gland development. Dev Biol 2009;328:188-199.

-6 Zhou W, Boucher RC, Bollig F, et al: Characterization of mesonephric development and regeneration using transgenic zebrafish. Am J Physiol Renal Physiol 2010;299:F1040F1047.

7 von Gise A, Zhou B, Honor LB, et al: WT1 regulates epicardial epithelial to mesenchymal transition through beta-catenin and retinoic acid signaling pathways. Dev Biol 2011; 356:421-431.

8 Kimmel CB, Ballard WW, Kimmel SR, et al: Stages of embryonic development of the zebrafish. Dev Dyn 1995;203:253-310.
9 DiBella LM, Park A, Sun Z: Zebrafish Tsc1 reveals functional interactions between the cilium and the TOR pathway. Hum Mol Genet 2009; 18:595-606.

10 Ryu YK, Collins SE, Ho HY, et al: An autocrine Wnt5a-Ror signaling loop mediates sympathetic target innervation. Dev Biol 2013;377:79-89.

11 Yamaguchi TP, Bradley A, McMahon AP, et al: A Wnt5a pathway underlies outgrowth of multiple structures in the vertebrate embryo. Development 1999;126:1211-1223.

12 Choi SY, Chacon-Heszele MF, Huang L, et al: Cdc42 deficiency causes ciliary abnormalities and cystic kidneys. J Am Soc Nephrol 2013; 24:1435-1450.

13 Siggberg L, Peippo M, Sipponen M, et al: 9q22 deletion - first familial case. Orphanet J Rare Dis 2011;6:45.

14 Tufan F, Cefle K, Turkmen S, et al: Clinical and molecular characterization of two adults with autosomal recessive Robinow syndrome. Am J Med Genet A 2005;136:185-189.

15 Qian D, Jones C, Rzadzinska A, et al: Wnt5a functions in planar cell polarity regulation in mice. Dev Biol 2007;306:121-133.

16 Luyten A, Su X, Gondela S, et al: Aberrant regulation of planar cell polarity in polycystic kidney disease. J Am Soc Nephrol 2010;21: 1521-1532.

17 Kramer-Zucker AG, Olale F, Haycraft CJ, et al: Cilia-driven fluid flow in the zebrafish pronephros, brain and Kupffer's vesicle is required for normal organogenesis. Development 2005;132:1907-1921.
18 Gao CL, Wang GL, Amack JD, et al: Oda16/ $\mathrm{Wdr69}$ is essential for axonemal dynein assembly and ciliary motility during zebrafish embryogenesis. Dev Dyn 2010;239:21902197.

19 Lunt SC, Haynes T, Perkins BD: Zebrafish ift57, ift 88 , and ift172 intraflagellar transport mutants disrupt cilia but do not affect hedgehog signaling. Dev Dyn 2009;238:1744-1759.

20 Schuchardt A, Dagati V, Larssonblomberg L, et al: Defects in the kidney and enteric nervous-system of mice lacking the tyrosine kinase receptor ret. Nature 1994;367:380-383.

21 Nishita M, Qiao S, Miyamoto M, et al: Role of Wnt5a-Ror2 signaling in morphogenesis of the metanephric mesenchyme during ureteric budding. Mol Cell Biol 2014;34:3096-3105.

22 Yun K, Ajima R, Sharma N, et al: Non-canonical Wnt5a/Ror2 signaling regulates kidney morphogenesis by controlling intermediate mesoderm extension. Hum Mol Genet 2014 Epub ahead of print.

-23 Levinson RS, Batourina E, Chol C, et al Foxd1-dependent signals control cellularity in the renal capsule, a structure required for normal renal development. Development 2005; 132:529-539.

24 Mendelsohn C, Batourina E, Fung S, et al: Stromal cells mediate retinoid-dependent functions essential for renal development. Development 1999;126:1139-1148. 